OPEN ACCESS

Edited by:

Loren Martin,

University of Toronto, Canada

Reviewed by:

Wiebke Gandhi,

University of Reading, United Kingdom

Kai Karos,

Open University of the

Netherlands, Netherlands

*Correspondence:

Lukas D. Linde

lukas.linde@ubc.ca

†These authors share senior authorship

Specialty section

This article was submitted to

Pain Research Methods,

a section of the journal

Frontiers in Pain Research

Received: 23 June 2021 Accepted: 06 December 2021 Published: 23 December 2021

Citation:

McDougall JF, Bailey NGN, Banga R, Linde LD and Kramer JLK (2021) The

Influence of Examiner Gender on

Responses to Tonic Heat Pain

Assessments: A Preliminary

Investigation

Front. Pain Res. 2:729860

doi: 10.3389/fpain.2021.729860

\section{The Influence of Examiner Gender on Responses to Tonic Heat Pain Assessments: A Preliminary Investigation}

\author{
Jessica F. McDougall ${ }^{1,2}$, Nicole G. N. Bailey ${ }^{1}$, Rohan Banga ${ }^{1}$, Lukas D. Linde ${ }^{1,3,4 *+}$ and \\ John L. K. Kramer ${ }^{1,3,4 \dagger}$ \\ ${ }^{1}$ International Collaboration on Repair Discoveries, University of British Columbia, Vancouver, BC, Canada, ${ }^{2}$ Department of \\ Rehabilitation Sciences, Faculty of Medicine, University of British Columbia, Vancouver, BC, Canada, ${ }^{3}$ Department of \\ Anesthesiology, Pharmacology and Therapeutics, Faculty of Medicine, University of British Columbia, Vancouver, BC, \\ Canada, ${ }^{4}$ Djavad Mowafaghian Centre for Brain Health, University of British Columbia, Vancouver, BC, Canada
}

Background: The influence of examiner gender on pain reporting has been previously explored in both research and clinical settings. However, previous investigations have been limited, with the majority of studies employing single, static assessments of pain (e.g., cold pressor test, verbal pain ratings). The impact of examiner gender on both static and dynamic heat-based pain assessments is currently unknown.

Methods: Thirty eight participants (20 females aged $24.1 \pm 4.44$, and 18 males, aged $24.8 \pm 4.54$ ) completed two identical testing sessions, randomized to a male and female examiner in a cross-over design. Pain sensitivity was examined using heat pain thresholds, verbal pain ratings to tonic heat, computerized visual analog scale (CoVAS) rating to tonic heat, and participant-controlled temperature (PCT) heat pain assessments.

Results: Female participants reported higher verbal pain to tonic heat with a female examiner compared to male participants, with similar trends for CoVAS responses to tonic heat. Conversely heat pain thresholds and PCT were not significantly influenced by experimenter gender.

Conclusions: Overall, verbal ratings were the most impacted by examiner gender, with temperature-based methods such as PCT and pain thresholds showing little to no examiner gender effects. While the gender of the examiner may be an important consideration in the measurement of sex and gender differences in pain research, the choice of pain assessment method may be of similar consequence.

Keywords: quantitative sensory testing, sex differences, gender differences, participant-controlled temperature, thermal pain

\section{INTRODUCTION}

The role of sex and gender on pain has been the source of substantial scientific and public discourse (1-5). In clinical settings, females experience acute and chronic pain with more frequency and to a greater intensity compared to males (2). Experimental studies employing pain sensitivity quantitative sensory testing [QST; a 
battery of tests which examines noxious and non-noxious somatosensory sensitivity (6)] outcomes (e.g., cold pressor tests, pain pressure thresholds) have provided complimentary support that females may be more sensitive to noxious stimuli than males $(2,4)$. Heterogeneity among these QST outcomes are commonplace, however, challenging the notion of the aforementioned robust sex or gender-related differences in pain perception (1).

A number of social factors have been proposed to contribute to variation in QST outcomes between experimental pain studies. These include individual and interpersonal factors, as well as environmental factors such as time of day $(7,8)$. Related to interpersonal factors, the social, gender context of the pain experience appears to influence pain report. Opposing examiner gender effects have been reported, with male participants tending to verbally report significantly less pain in the presence of a female examiner (and vice versa for female participants) $(7,9,10)$ [note: gender is used in this regard given that these effects are social as opposed to biological (5)]. This follows the Gender Context Model of Pain, which suggests men will be less likely to express pain, especially if the examiner is perceived as being threatening to masculine gender roles, whereas women will be more likely to express pain. However, this difference may be dependent on how pain is expressed. Verbal pain report may be more susceptible to these gender differences than non-verbal expressions $(11,12)$. Indeed, individual factors add complexity. One possible explanation for these reported gender specific examiner effects may be differences in catastrophizing - a negative cognitive-affective response to pain (13). Catastrophizing is associated with increased pain across a variety of pain measures and may be influenced by the presence of others $(4,13)$. Moreover, sex differences in catastrophizing have been reported, insofar as women tend to catastrophize more than men (4). As such, catastrophizing may also modulate the interaction between sex and social interaction of pain measurements.

A major limitation of previous experimenter/participant gender investigations has been a narrow focus on pain tolerance, measured chiefly by way of the cold pressor test (7). Advances in QST techniques have led to the development of various static and dynamic outcomes, which have been widely employed to investigate sex/gender differences in pain perception (14). Painful thermal dynamic and static QST measures have shown significant differences between male and female participants (4) and may be differently susceptible to experimenter gender influence, and to gender stereotypes. For example, verbal pain ratings of heat pain involve direct verbal communication with experimenters in response to a noxious stimulus, conversely, automated metrics of pain assessment, such as participant controlled temperature (PCT) (15), require less direct communication with examiners. Verbal pain report has been shown to be susceptible to the gender context in which the report occurs $(7,9,10)$, however it is not known how susceptible PCT-a non-verbal form of pain expression-is to these gendered influences. It stands to reason that such differences in participant/experimenter interactions within QST assessments may influence the effect of experimenter gender on pain perception. Including both verbal and non-verbal pain reports to both a male and female examiner allows us to tease apart the impact of social context on the apparent sex/gender differences in pain. To our knowledge, no previous studies have explored the influence of experimenter gender on pain outcomes assessed using multiple painful heat QST techniques.

Our aim was to determine the extent to which modern QST heat-pain measures are influenced by the gender of the examiner. To this end, we employed verbal and non-verbal rating and temperature-based (non-verbal) methods of reporting sensitivity to heat pain, with both static and dynamic outcomes. A secondary, exploratory aim explored role of psychosocial factors, specifically the effect of pain catastrophizing on experimenter gender effects on pain outcomes. We expected to see greater gender differences in verbal ratings-based measurements of pain compared to temperature-based measurements, such that males would verbally rate pain as lower in the presence of a female examiner, and females would demonstrate opposite and smaller effect. We anticipate temperature-based methods to show smaller or non-significant effect, as these rely on less direct social interaction during pain reporting.

\section{MATERIALS AND METHODS}

\section{Participants}

We determined 40 participants (females aged $24.1 \pm 4.44$, and males, aged $24.8 \pm 4.54$ ) would provide a partial eta-squared $\left(\eta_{\mathrm{p}}^{2}\right)=0.05$, with a power of 0.8 and an alpha of 0.5 (calculation completed in $G^{*}$ Power 3.1) (16). This $\eta_{\mathrm{p}}^{2}$ was estimated from previous studies that have compared the interaction of experimenter and participant gender on pain outcomes (1719). Exclusion criteria included presence or history of chronic pain (i.e., pain persisting longer than 3 months), determined from a self-reported health history questionnaire. All participants were over 18 years of age and provided informed consent. Participants were recruited from the local university and hospital communities through flier advertisements.

\section{Experimenters}

The experimenters were a cis-female aged 22 and a cis-male aged 19. Both wore a lab coat over jeans and a shirt, and both identified as cis-gendered [i.e., indicated that their gender (man/woman) did not differ from their sex (male/female)]. We did not control for other experimenter characteristics (e.g., height, weight, or race), and these characteristics were not collected from participants. Scripts were created to standardize interactions with the participants, including instructions for all pain tests.

\section{Procedure}

Participants were randomly assigned to a male or female examiner on day 1 in a counterbalanced design, such that half of the participants began with the male examiner, while the other half began with the female examiner (Figure 1). Sessions were at least $24 \mathrm{~h}$ apart. Each testing day was designed to be approximately 1-h long. The true nature of the study was withheld from participants, who were led to believe that the purpose was to compare two measures of testing heat pain. Given 


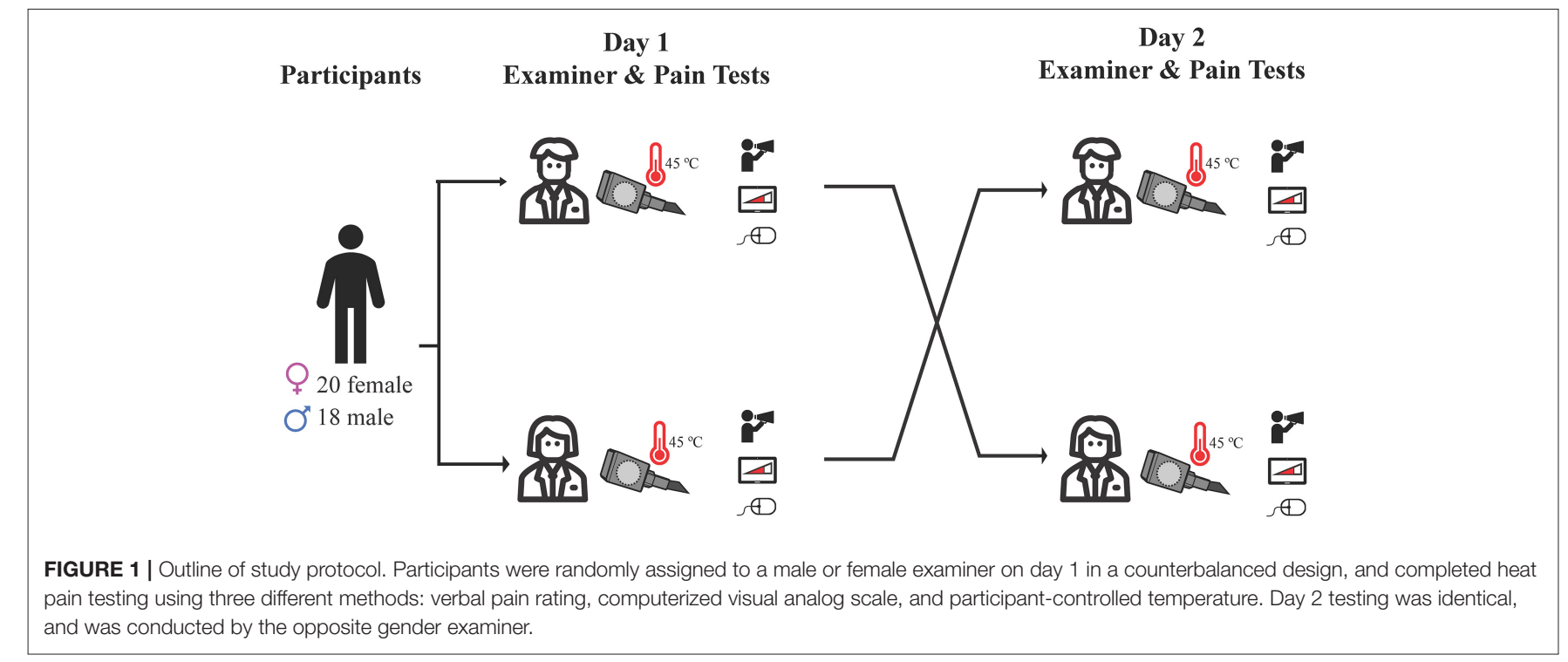

the blinded nature of our study, the experimenter followed a script that introduced them as the research assistant for the study, with no mention of their gender or the true nature of the study. Experimenters stayed close beside the participant for all tests, standing beside the participant and alternating between watching a computer screen (where the test results were being shown), making an arbitrary note on a clipboard, and glancing at the participant to ensure protocols were being followed. The switch of experimenters was explained to participants as a "scheduling conflict," and the other experimenter was filling in due to the absence. At the end of day 2, participants were fully debriefed. This involved the experimenter outlining the need for deception and offering participants the opportunity to withdraw their data from the study. All participants were then asked if they suspected or knew the true purpose of the study. All study procedures were approved by the Behavioral Research Ethics Board at the University of British Columbia (approval number H1900944), and were conducted in accordance with the Declaration of Helsinki (20) involving research on human participants. Our study protocol was not pre-registered, due the required deception of participants (i.e., pre-registering planned statistical comparisons could give away the true nature of the study).

\section{Heat Pain Measurements}

Heat pain thresholds and responses to prolonged heat pain were performed using a calibrated thermode (Medoc Advanced Medical Systems, Ramat Yishai, Israel, CHEPs thermode, $27 \mathrm{~mm}$ diameter) applied on the palmar aspect of the forearm. Heat pain thresholds were performed first on the distal $1 / 3$ of a randomly chosen forearm, followed by either PCT or continuous visual analog scale (CoVAS) heat tests performed on the proximal $2 / 3$ of the same forearm-the order of the PCT and CoVAS test presentation was randomized. Prior to the presentation of each heat test, a familiarization test took place to introduce participants to the pain-rating method. A 5-min break separated the three tests (heat pain thresholds, familiarization, and tonic heat test). Following the first tonic heat test, a 10 -min break took place. Heat pain thresholds were then performed on the distal 1/3 of the other forearm, followed by the PCT or CoVAS, whichever was randomized to be performed second. Another familiarization test was performed prior to the introduction of the second tonic heat test. Again a 5-min break separated each of the three tests (heat pain thresholds, familiarization, and tonic heat test).

\section{Heat Pain Thresholds}

For heat pain thresholds, the thermode temperature was increased at a rate of $1^{\circ} \mathrm{C} / \mathrm{s}$ from a baseline of $32^{\circ} \mathrm{C}$ to a maximum of $55^{\circ} \mathrm{C}$. Participants were instructed to press a button when the first sensations of pain were perceived (i.e., when the original impression of warmth or heat turned into the feeling of "burning," "stinging," "aching," or "drilling”) (6). Upon button press, the heat thermode returned to the baseline temperature of $32^{\circ} \mathrm{C}$ at a rate of $70^{\circ} \mathrm{C} / \mathrm{s}$. Four trials were conducted consecutively with at least $5 \mathrm{~s}$ between each trial. The main outcome measure from pain threshold assessments was the average temperature of the initial pain sensations over the four trials.

\section{Tonic Heat Pain}

Participants continuously rated their pain perception throughout a 2-min application of tonic heat $\left(45^{\circ} \mathrm{C}\right)$ via CoVAS (Medoc Advanced Medical Systems, Ramat Yishai, Israel). The initial temperature of the thermode increased at a rate of $70^{\circ} \mathrm{C} / \mathrm{s}$, and reached $45^{\circ} \mathrm{C}$ from a baseline of $32^{\circ} \mathrm{C}$, then was maintained at $45^{\circ} \mathrm{C}$ for $2 \mathrm{~min}$ of tonic heat. We chose $45^{\circ} \mathrm{C}$ for tonic heat pain to maintain similar sensations to the participantcontrolled temperature assessment described below (15). At the end of the $2 \mathrm{~min}$, participants also reported their pain verbally to the experimenter (0-10, 0- "no pain at all," 10- "worst pain imaginable"). Participants were instructed to rate their pain using a slider on the CoVAS machine, which has a visual of a linear increasing graph, indicating no pain on one end and the maximal amount of pain they could tolerate on the opposite end. 
Participants were asked to rate their pain continuously, moving the slider as desired. The rating was recorded every $20 \mathrm{~ms}$. The average pain rating from the CoVAS readings was recorded as average pain rating to tonic heat.

\section{Participant Controlled Temperature}

For participant controlled temperature (PCT), participants continuously adjusted the temperature of the thermode to maintain their initial perception (15). For example, if at the beginning of the 2-min trial $\left(\right.$ at $45^{\circ} \mathrm{C}$ ) participants rated the pain as a $4 / 10$, they were instructed to either increase or decrease the temperature in order to maintain the $4 / 10$ sensation over the $2 \mathrm{~min}$. Participants were provided a computer mouse to control temperature, whereby left and right button clicks changed the temperature by $\pm 0.1^{\circ} \mathrm{C}$, respectively. Participants were informed that the temperature "may feel as though it is increasing or decreasing," and were asked to maintain their initial perception by raising or lowering the heat through clicking the mouse. To confirm participants maintained their pain rating throughout the $2 \mathrm{~min}$, each was asked to verbally report their pain at the beginning and end of the protocol. The protocol was identical to that presented by Jutzeler et al. (15). Average temperature across the $2 \mathrm{~min}$ of PCT was taken as the primary outcome.

\section{Familiarization to Heat Pain Assessments}

Familiarization trials for both CoVAS and PCT were conducted on a neutral test site. Participants were exposed to 1 min of heat, beginning at a baseline of $40^{\circ} \mathrm{C}$. Then, the temperature oscillated by $\pm 2^{\circ} \mathrm{C}$ at rate of $0.5^{\circ} \mathrm{C} / \mathrm{s}$. During this time, participants were instructed to rate their pain for CoVAS or to maintain consistent pain sensations via button clicks for PCT. This oscillation in temperature provided participants the opportunity to become accustomed to both heat sensations and the CoVAS and PCT apparatus in response to multiple temperatures. The familiarization trials also helped to reinforce the concept that the temperature in the PCT trials also could be perceived as though it was increasing or decreasing, supporting the blinding of participants to the nature of the PCT trials.

\section{Questionnaires}

At the conclusion of the second day of testing, the pain catastrophizing scale (PCS) questionnaire was administered. The PCS involves the participant rating 13 statements regarding the types of thoughts and feelings that occur when they are in pain from 0 ("not at all") to 4 ("all the time"). There are three subscales in the PCS; magnifying (three items, "I become afraid that the pain will get worse"), rumination (four items, "I keep thinking about how badly I want the pain to stop"), and helplessness (six items, "It's terrible and I think it's never going to get any better"). Higher PCS scores have been associated with greater levels of pain and pain-focused experiences (21). PCS scores also tend to be higher in females (1).

A demographics questionnaire was also delivered on the first day of testing, asking participants to report their sex, gender, and age. For gender, participants were asked "What is your gender?" with options for "female," "male," "non-binary/third gender," "prefer to self describe," or "prefer not to say."

\section{Statistical Analysis}

Cohen's d effect sizes were calculated for differences in pain outcomes between male and female participants separately by examiner. This was done to simply model pain outcomes measured by a single examiner of one sex, as would be commonplace in previous studies. The primary outcomes were verbal pain rating following 2 min of tonic heat, average CoVAS rating over $2 \mathrm{~min}$ of tonic heat pain, heat pain thresholds, and average temperature over $2 \mathrm{~min}$ of PCT assessment. Descriptive statistics were assessed using histograms, box plots, and QQ plots to confirm normal distributions of pain outcomes. A preliminary analysis revealed that all pain outcomes were normally distributed (Shapiro-Wilk test range: 0.05-0.29). To formally and comprehensively test our study design, we adopted a repeated measure ANOVAs approach with participant gender as a between-subject variable, and examiner gender as the withinsubject variable. Order of testing (i.e., day 1 or day 2) was considered as a covariate to confirm effects were due to the examiner gender and not the repeat-testing nature of the study design. Significant interaction effects were further explored with post hoc Bonferroni corrected pairwise comparisons.

Relationships between PCS and pain outcomes were explored using bivariable Pearson correlations, with a Bonferroni correction for multiple comparisons. We examined relationships between pain scores and PCS across both testing sessions as well as explored associations between PCS scores and relative differences in pain scores between testing sessions (i.e., examiners).

\section{RESULTS}

Forty participants were recruited, 38 of which completed both sessions (20 females and 18 males). Missing data from the two subjects was due to technical issues with the heat stimulator-they were unable to complete either day of testing. All other subjects completed both experimental sessions. No subjects withdrew their data after debriefing. Upon debrief, all participants confirmed no knowledge of the true purpose of the study. All participants identified as cis-gendered.

\section{Rating Based Methods}

There was a significant main effect of participant gender on verbal pain rating to tonic heat $\left[F(1,36)=5.77, p=0.02, \eta_{\mathrm{p}}^{2}=\right.$ $0.14]$. This suggests that female participants verbally reported heat as more painful than men. Examiner gender had no main effect on verbal pain ratings $\left[F(1,36)=0.93, p=0.34, \eta_{p}^{2}=0.03\right]$. However, there was a significant interaction effect for participant and experimenter gender on verbal pain rating $\left[F_{(1,36)}=5.61\right.$, $\left.p=0.02, \eta_{\mathrm{p}}^{2}=0.14\right]$. Bonferroni corrected post-hoc analysis revealed that female participants verbally reported higher tonic heat pain than males in the presence of a female examiner $(t$ $=3.21, p=0.01)$. Order of day of testing did not influence the gender effect $\left[F_{(1,36)}=0.01, p=0.91\right]$. For average CoVAS ratings, there were no significant main effects of participant $\left[F_{(1,36)}=1.20, p=0.28, \eta_{\mathrm{p}}^{2}=0.03\right]$ or examiner gender $\left[F_{(1,36)}\right.$ $\left.=3.88, p=0.06, \eta_{\mathrm{p}}^{2}=0.10\right]$. There was also no significant 

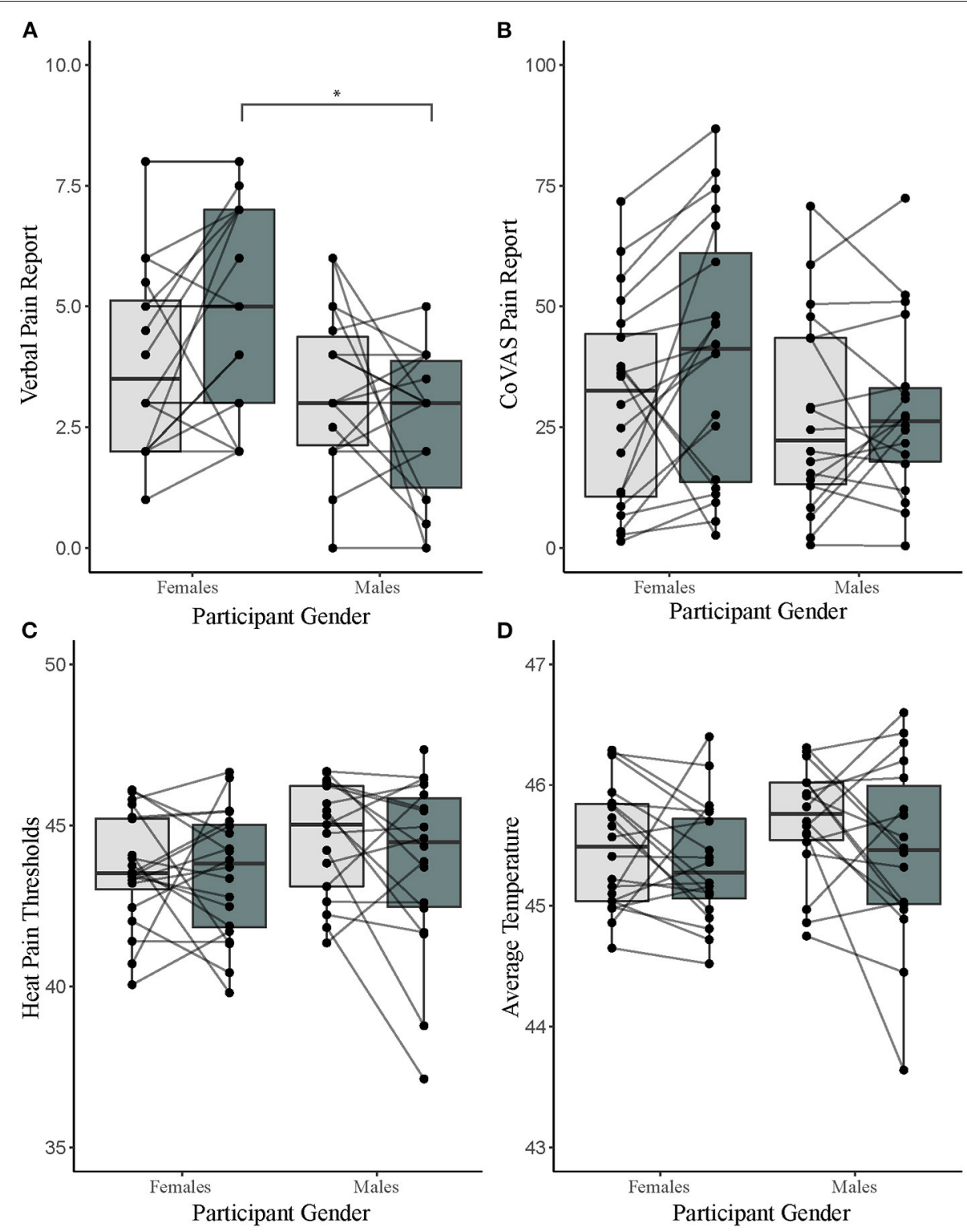

Examiner 官 Male 官 Female

FIGURE 2 | Results of pain tests separated by participant and examiner gender. (A) Verbal pain reports for a 2-min tonic heat test separated by gender. (B) computerized visual analog scale (CoVAS) reports for a 2-min tonic heat test separated by gender. (C) Average heat pain threshold reports separated by gender. (D) PCT reports (as calculated by average temperature over a for a 2-min tonic heat test) separated by gender. *denotes significance level of $p<0.05$ from Bonferroni corrected post hoc analysis.

interaction effect $\left[F_{(1,36)}=2.70, p=0.11, \eta_{\mathrm{p}}^{2}=0.07\right]$ (Figure 2; Table 1). Order of session did not influence CoVAS ratings $\left[F_{(1,36)}=3.08, p=0.09\right]$.

\section{Temperature Based Methods}

There was no significant main effect of participant [heat pain thresholds: $F_{(1,36)}=1.80, p=0.19, \eta_{\mathrm{p}}^{2}=0.05$; PCT: $F_{(1,36)}=1.02$, $p=0.32, \eta_{\mathrm{p}}^{2}=0.03$ ] or examiner gender [heat pain thresholds: $F_{(1,36)}=2.64, p=0.11, \eta_{\mathrm{p}}^{2}=0.07$;CT: $F_{(1,36)}=3.31, p=$ $\left.0.08, \eta_{\mathrm{p}}^{2}=0.08\right]$. There was also no significant interaction effect between participant and examiner gender [heat pain thresholds: $F_{(1,36)}=1.59, p=0.22, \eta_{\mathrm{p}}^{2}=0.04$; PCT: $F_{(1,36)}=0.45, p=0.51$, $\left.\eta_{\mathrm{p}}^{2}=0.01\right]$ (Figure 2; Table 1). Order of session did not influence PCT scores $\left[F_{(1,36)}=0.56, p=0.46\right]$ or pain thresholds $\left[F_{(1,36)}=\right.$ $0.66, p=0.42]$.

We also ran a repeat measures ANOVA on the initial rating of the PCT stimulus to investigate if there was a gender difference in this initial perception. There was no significant difference between genders $\left[F_{(1,36)}=2.417, p=0.129\right]$, nor was there an 


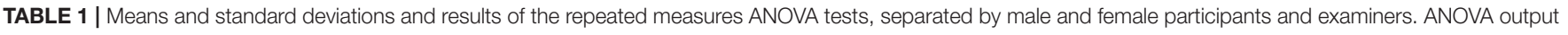
for interaction effect presented.

\begin{tabular}{|c|c|c|c|c|c|}
\hline & \multicolumn{2}{|c|}{$\begin{array}{c}\text { Female examiner } \\
\text { Mean (SD) }\end{array}$} & \multicolumn{2}{|c|}{$\begin{array}{c}\text { Male examiner } \\
\text { Mean (SD) }\end{array}$} & \multirow{2}{*}{$\frac{\text { RM-ANOVA }^{\mathrm{a}}}{\boldsymbol{F}_{(\boldsymbol{p})}}$} \\
\hline & Females & Males & Females & Males & \\
\hline PCT & $45.36(0.49)$ & $45.48(0.51)$ & $45.45(0.75)$ & $45.70(0.47)$ & $0.45(0.51)$ \\
\hline CoVAS & $29.83(21.23)$ & $40.34(26.25)$ & $28.45(18.16)$ & $27.50(20.53)$ & $2.70(0.11)$ \\
\hline Heat pain thresholds & $43.64(1.73)$ & $43.5(1.97)$ & $43.84(2.74)$ & $44.93(2.32)$ & $1.59(0.22)$ \\
\hline Verbal rating & $4.00(2.03)$ & $4.93(2.25)$ & 3.33 (1.64) & $2.94(1.52)$ & $5.61(0.02)$ \\
\hline
\end{tabular}

${ }^{a} d f=19$, interaction effect of participant gender $x$ examiner gender.

RM-ANOVA, repeated measure ANOVA; PCT, participant controlled temperature; CoVAS, computerized visual analog scale.

TABLE 2 | Correlations coefficients (R) between pain catastrophizing subscales and pain measurements adjusted for multiple comparisons (Bonferroni).

\begin{tabular}{|c|c|c|c|c|c|c|c|c|c|c|c|c|}
\hline & \multicolumn{4}{|c|}{ Male examiner } & \multicolumn{4}{|c|}{ Female examiner } & \multicolumn{4}{|c|}{$\begin{array}{l}\text { Difference scores between } \\
\text { male and female examiners }\end{array}$} \\
\hline & CoVAS & PCT & PT & Verbal & CoVAS & PCT & PT & Verbal & CoVAS & PCT & PT & Verbal \\
\hline Rumination & 0.16 & -0.16 & -0.23 & 0.12 & 0.10 & -0.23 & -0.16 & 0.22 & 0.05 & 0.10 & -0.05 & -0.13 \\
\hline Magnification & 0.14 & -0.14 & -0.21 & 0.01 & 0.09 & -0.05 & -0.11 & 0.08 & 0.05 & -0.06 & -0.08 & -0.09 \\
\hline Helplessness & 0.23 & -0.32 & -0.28 & 0.20 & 0.20 & -0.03 & -0.09 & 0.28 & 0.00 & -0.22 & -0.16 & -0.13 \\
\hline Total & 0.21 & -0.24 & -0.27 & 0.14 & 0.16 & -0.11 & -0.13 & 0.23 & 0.03 & -0.08 & -0.11 & -0.13 \\
\hline
\end{tabular}

effect of examiner gender $\left[F_{(1,36)}=1.490, p=0.230\right]$. Average initial rating for female participants was $5.55 \pm 1.56$ when tested by the male examiner and $5.80 \pm 1.64$ when tested by the female examiner. Average initial rating for male participants was $4.83 \pm$ 1.58 when tested by the male examiner and $5.00 \pm 1.57$ when tested by the female examiner. Additionally, 33/38 participants reported the same pain rating at the beginning and end of the PCT test, 4 were within $\pm 1 / 10$ on an NRS, and 1 participant was within $\pm 2 / 10$ on an NRS. This is in contrast to the CoVAS test, where the range was $\pm 3 / 10$ on the NRS.

\section{PCS Correlations to Pain Outcomes}

PCS subscales were not correlated to any pain outcomes in both males and females, and were also not correlated to relative difference in pain outcomes between examiners (Table 2).

\section{DISCUSSION}

The impact of examiner characteristics on study outcomes have been attributed a causal role in the ongoing scientific replication crisis (3). Among concerns is that the gender of the examiner contributes to heterogeneous outcomes between studies. As predicted by the Gender Context Model of Pain, we observed that sex differences in tonic heat pain perception may be exaggerated by verbal rating-based methods when the examiner is female. CoVAS pain ratings demonstrated similar trends, albeit not significant. In contrast, temperature-based methods of assessing heat pain were not significantly affected by the gender of the examiner.
To our knowledge, the effect of examiner gender on pain outcomes has been explicitly tested in six previous studies (see Table 3 for description) (9, 10, 17-19, 22). For subjective pain ratings, our observations correspond with those reporting an opposing examiner gender effect $(9,10,17,18)$ as well as social theories of pain which propose the gender context in which pain is expressed influences pain report (12). The former was evidenced in our reported verbal ratings in women, which were significantly higher in the presence of a female compared to a male examiner. Similar, albeit more variable results were observed for CoVAS ratings to heat pain. Our findings support the notion that pain communication may be more affected by gender interactions as compared to the actual pain experience. For example, when comparing verbal pain ratings to CoVAS ratings, the pain experience (CoVAS) was comparable, while the act of reporting to the experimenter verbally was influenced my experimenter gender. The notion that pain communication, but not experience, is influenced by gender is supported by a previous study that showed biological responses to pain (e.g., autonomic changes) are unaffected by examiner gender (17). Taken together, our findings provide evidence for a dissociation between pain experience and pain reporting, which is influenced by examiner gender. Overall, this lends support to the Gender Context Model of Pain (12), in that outcomes with the most social communication were more influenced by experimenter gender.

The modernization of QST assessments has seen a shift to temperature-based methods, including standardized methods of measuring heat pain thresholds (6). Previous studies exploring experimenter gender effects (Table 3) have not incorporated temperature-based methods of assessing pain, relying instead on 
TABLE 3 | Summary of studies examining the effect of examiner gender on pain outcomes.

\begin{tabular}{|c|c|c|c|c|c|}
\hline References & Test stimuli & Rating method & Gender effects & Additional measures & Study design \\
\hline $\begin{array}{l}\text { Levine and De } \\
\text { Simone (9) }\end{array}$ & $\begin{array}{l}\frac{\text { Cold pressor }}{\text { Both hands in }} \\
0-1^{\circ} \mathrm{C} \text { ice bucket }\end{array}$ & $\begin{array}{l}\text { Pain intensity } \\
\text { Numeric rating scale, given } \\
\text { every } 15 \mathrm{~s} \text { for } 180 \mathrm{~s}\end{array}$ & $\begin{array}{l}\text { Intensity } \\
\text { Male participants reported } \\
\text { lower pain intensity to a } \\
\text { female experimenter }\end{array}$ & $\begin{array}{l}\text { Pain Affective scale } \\
\text { - Males reported less } \\
\text { negative affective words } \\
\text { to female experimenter }\end{array}$ & $\begin{array}{l}\text { Parallel experimental design } \\
\text { Participants } \\
n=68 \text { (33 female, } 35 \text { male) } \\
\text { Ages 17-29 }(\mathrm{M}=19.13)\end{array}$ \\
\hline Kallai et al. (18) & $\begin{array}{l}\text { Cold pressor } \\
\text { Non-dominant } \\
\text { hand in circulating } \\
-1^{\circ} \mathrm{C} \text { ice bucket }\end{array}$ & $\begin{array}{l}\text { Pain intensity } \\
\text { 10-point rating scale, given } \\
\text { immediately after CPT } \\
\text { Pain threshold } \\
\text { Seconds } \\
\text { Pain tolerance } \\
\text { Seconds }\end{array}$ & $\begin{array}{l}\underline{\text { Intensity }} \\
\text { Both male and female } \\
\text { participants reported higher } \\
\text { pain intensity to a female } \\
\text { experimenter } \\
\text { Tolerance } \\
\text { Female participants had } \\
\text { higher pain tolerances with } \\
\text { a male experimenter Male } \\
\text { participants had higher pain } \\
\text { tolerances with a } \\
\text { female experimenter } \\
\text { Threshold } \\
\text { No experimenter gender } \\
\text { effect found for } \\
\text { pain threshold }\end{array}$ & $\begin{array}{l}\text { Participants rated the } \\
\text { examiner's authority, } \\
\text { competence, likeability and } \\
\text { masculinity/femininity on } \\
\text { seven-point rating scales }\end{array}$ & $\begin{array}{l}\text { Parallel experimental design } \\
\text { Participants } \\
n=160 \text { (80 female, } \\
80 \text { male) Female ages } \\
17-36(\mathrm{M}=23.19, \mathrm{SD} 3.59) \\
\text { Male ages 19-59 (M= } \\
\text { 24.55, SD 5.79) }\end{array}$ \\
\hline $\begin{array}{l}\text { Gijsbers and } \\
\text { Nicholson (10) }\end{array}$ & $\begin{array}{l}\text { Pressure } \\
\text { Pressure } \\
\text { algometer with } \\
0-9 \mathrm{~kg} \text { force range } \\
\text { on upper sternum }\end{array}$ & $\frac{\text { Pain threshold }}{\text { kilograms }}$ & $\begin{array}{l}\text { Threshold } \\
\text { Male participants had higher } \\
\text { pain thresholds with a } \\
\text { female examiner }\end{array}$ & $\begin{array}{l}\text { Anxiety } \\
\text { - Measured with } 10 \mathrm{~cm} \\
\text { VAS } \\
\text { - Anxiety was low for both } \\
\text { female and male } \\
\text { participants } \\
\text { - No correlation with } \\
\text { pain thresholds } \\
\text { McGill Pain Questionnaire } \\
\text { - No significant examiner } \\
\text { gender effect on pain } \\
\text { scores } \\
\text { - Indicated low emotional } \\
\text { concern in participants }\end{array}$ & $\begin{array}{l}\text { Parallel experimental design } \\
\text { Participants } \\
n=64 \text { (32 females, } \\
32 \text { males) Female ages } \\
\text { 18-36 (M = 21.0, SD 4.4) } \\
\text { Male ages 18-49 }(\mathrm{M}=23.0, \\
\text { SD 8.1) }\end{array}$ \\
\hline Weisse et al. (19) & $\begin{array}{l}\text { Cold pressor } \\
\text { Non-dominant } \\
\text { hand in } 0-2^{\circ} \mathrm{C} \\
\text { ice bucket }\end{array}$ & $\begin{array}{l}\text { Pain intensity } \\
0-20 \text { rating scale every } 15 \mathrm{~s} \\
\text { for a total of } 300 \mathrm{~s}\end{array}$ & $\begin{array}{l}\text { Intensity } \\
\text { No main effect found for } \\
\text { pain reporting and examiner } \\
\text { gender. However, an } \\
\text { interaction was found with } \\
\text { participant race and } \\
\text { examiner gender: Black } \\
\text { participants reported higher } \\
\text { pain intensities than white } \\
\text { participants to a } \\
\text { female examiner }\end{array}$ & $\begin{array}{l}\text { Pain unpleasantness scale } \\
\text { - No main effect for pain } \\
\text { reporting and examiner } \\
\text { gender } \\
\text { - An interaction found for } \\
\text { participant race and } \\
\text { examiner gender: black } \\
\text { participants reported } \\
\text { more unpleasantness } \\
\text { than white participants to } \\
\text { a female examiner }\end{array}$ & 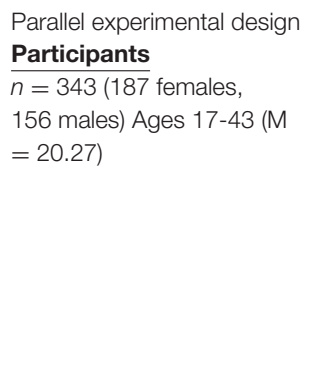 \\
\hline Aslaksen et al. (17) & $\begin{array}{l}\text { Heat TSA II } \\
\text { Neurosensory } \\
\text { Analyzer (Medoc, } \\
\text { Israel): } 30 \bullet 30 \mathrm{~mm} \\
\text { aluminium contact } \\
\text { thermode with a } \\
10^{\circ} \mathrm{C} / \mathrm{s} \text { change } \\
\text { rate on } \\
\text { right forearm }\end{array}$ & $\begin{array}{l}\frac{\text { Pain intensity }}{100 \mathrm{~mm} \text { VAS }} \\
\text { Physiological } \\
\text { pain response } \\
\text { Heartrate variability and skin } \\
\text { conductance levels }\end{array}$ & $\begin{array}{l}\text { Intensity } \\
\text { Male participants reported } \\
\text { lower pain intensity to a } \\
\text { female examiner } \\
\text { Physiological pain response: } \\
\text { No examiner gender effect } \\
\text { found for } \\
\text { physiological responses }\end{array}$ & $\begin{array}{l}\text { Pain unpleasantness scale } \\
\text { - No significant examiner } \\
\text { gender effect } \\
\text { - Short } \\
\text { Adjective Check List } \\
\text { and Self-Assessment } \\
\text { Manikin scale } \\
\text { - Male participants } \\
\text { reported lower arousal to } \\
\text { female experimenters } \\
\text { - No significant examiner } \\
\text { gender effect with } \\
\text { subjective stress or } \\
\text { mood scales }\end{array}$ & $\begin{array}{l}\text { Parallel experimental design } \\
\text { Participants } \\
n=64 \text { (32 females, } \\
32 \text { males) Female ages } \\
\text { 19-40 (M = 23.61, SD 3.99) } \\
\text { Male ages 19-35 ( } M=23.3 \text {, } \\
\text { SD 2.49) }\end{array}$ \\
\hline
\end{tabular}


TABLE 3 | Continued

\begin{tabular}{|c|c|c|c|c|c|}
\hline References & Test stimuli & Rating method & Gender effects & Additional measures & Study design \\
\hline Vigil et al. (22) & $\begin{array}{l}\text { Cold pressor } \\
\text { One of two CPT } \\
\text { protocols used on } \\
\text { left hand: (1) } 5^{\circ} \mathrm{C} \\
\pm 1^{\circ} \mathrm{C} \text { circulating } \\
\text { ice bucket, or (2) } \\
\text { Isotemp 6200R28 } \\
\text { (Fisher Scientific, } \\
\text { USA) } \\
\text { electromechanical } \\
\text { CPT device at } 5^{\circ} \mathrm{C} \\
\pm 0.1^{\circ} \mathrm{C}\end{array}$ & $\begin{array}{l}\text { Pain intensity } \\
\text { 10-point VAS, } 30 \text { s into CPT } \\
\text { Pain threshold } \\
\text { Seconds } \\
\text { Pain tolerance } \\
\text { Seconds }\end{array}$ & $\begin{array}{l}\frac{\text { Intensity }}{\text { Both male and female }} \\
\text { participants reported higher } \\
\text { pain intensity to a female } \\
\text { examiner } \\
\frac{\text { Tolerance }}{\text { Subjects had higher pain }} \\
\text { tolerances with a } \\
\text { male examiner } \\
\text { Threshold } \\
\text { No examiner gender effect } \\
\text { found for pain threshold }\end{array}$ & $\begin{array}{l}\text { No additional measures } \\
\text { performed }\end{array}$ & $\begin{array}{l}\text { Parallel experimental design } \\
\text { Participants } \\
\frac{n=352(48 \% \text { males }) \text { Ages }}{18-30(\mathrm{M}=19.8, \mathrm{SD} 2.1)}\end{array}$ \\
\hline
\end{tabular}

CPT, cold pressor test.

verbal ratings or time-based approaches that assess tolerance (e.g., cold pressor). To address this limitation, we assessed examiner gender effects on pain threshold determined by method of limits and PCT. The latter, a revitalized approach based a method originally established by Hardy and Greene (23), involves participants continuously adjusting the temperature of the thermode over $2 \mathrm{~min}$ in order to maintain their initial perception of noxious heat (15). The concept of PCT is similar to CoVAS, but dynamic aspects of pain (i.e., the fluctuations in the perception of a constant painful stimuli over time) are reflected by changes in temperature as opposed to continuous ratings (15). Compared to CoVAS and verbal pain ratings, PCT provides pain reporting with the least obvious social context. Where verbal pain ratings involved direct communication with examiners and CoVAS involved the perceived communication of digital 0-10 scale, PCT involves button clicks to maintain sensation. To that end, PCT was more resilient to gender effects compared to verbal or CoVAS outcomes, as examiner gender did not significantly influence PCT. These findings provides further support for the social context of pain model, as PCT is less clearly a "rating" of pain to an examiner, and thus less influenced by the social context (12).

To consider a potential psychosocial factor, we aimed to explore the relationship between participants' PCS scores and variability introduced by the gender of the examiner. For pain catastrophizing, we observed no significant associations between PCS scores and any pain outcomes, for both raw scores as well as evaluating relative differences in pain outcomes between examiners. This suggests that pain catastrophizing does not have a significant influence on our observed gender effects on pain outcomes.

\section{Limitations}

Our findings are limited to a relatively homogenous population (i.e., undergraduate and graduate students). The extent our results are generalizable to other populations (e.g., older, community dwelling adults) requires further study. We also did not collect or report relationships between the race, ethnicity, height, or weight of our participants or examiners and the possible effects on pain ratings. This was beyond the scope of our current study and represents another avenue for further exploration. To that end, we did not control for experimenter ethnicity, or other examiner characteristics (e.g., hair color, eye color etc.). We sought to maintain ecological validity in our selection of a male and female examiner, rather than overly constrain various aspects of personal appearance/characteristics. To that end, our findings are based on the effect of clearly male and clearly female examiners.

In comparison to previous investigations of experimenter gender effects on pain perception $(9,10,17-19,22)$, our study is limited to a relatively small sample size. However, as a seminal study to explore experimenter gender effects on multiple heat-pain outcomes, our sample size was chosen pragmatically and in accordance with a sample size calculation related to quantitative pain assessments previously used in similar experimenter gender comparisons. We were unable to collect data on two participants due to technical issues, resulting in a fewer number of participants than reported in our a priori power calculation. We reported $\eta_{\mathrm{p}}^{2}$ values for all repeated measures ANOVA analyses along with Cohen's d values to highlight within experimenter effects. Finally, our findings are also limited to our included heat pain-based assessment methods. Future studies should continue to explore experimenter gender effects in other pain outcomes making use of differing modalities, such as mechanical pinpricks and more modern cold pain assessments.

We did not have our examiners conform to stereotypical gender roles, which may have muted examiner effects. Studies whose examiners dressed in "stereotypical gender conforming" ways $(9,10,17,18)$ appear more likely to see significant examiner effects compared to those that did not control for dress (2431). Status of the examiner may also matter-participants of both genders report higher pain tolerance to "high status" (i.e., professionally dressed, used formal names) examiners (18). In the present study we attempted to control for gender stereotypes through recruiting peer examiners that wore a uniform-lab coat over pants and a t-shirt-and that used the same script. This moderate "de-gendering" of the examiners and reduction of potential power imbalances through using peers may have reduced gender differences in the heat pain assessments.

Also, our study and those previous have focused on participants that conformed to gender norms. It is not clear if those who do not conform to gender norms may report 
pain differently or have different examiner-participant gender interaction effects in the reporting of pain. Examining pain in transgendered and non-binary individuals represents an important and understudied area of pain science-an area that would not only shed light on a marginalized populations' pain experience, but would also extend our understanding of the interaction between gender and pain.

\section{Conclusions and Future Implications}

Overall, our findings are aligned with the Gender Context Model of Pain, insofar as those outcome measures that were most likely to be influenced by social factors (i.e., verbal pain ratings) were more susceptible to experimenter gender effects, while outcomes less likely to be influenced by social factors (i.e., PCT) were not significantly influenced by experimenter gender. The examiner and participant gender can both influenced pain reporting, with the perceived level of examiner-participant interaction appearing to mediate these effects. Researchers should consider the social environment of their experiments, the pain measurement used, and the gender of their experimenters as these factors all play a role in detecting sex/gender differences in pain measurements. The use of non-verbal pain measures, with little to no examiner influence (e.g., coded temperature information via PCT) may be a potential solution to circumvent the effects of experimenter gender on pain related outcomes.

\section{REFERENCES}

1. Racine M, Tousignant-Laflamme Y, Kloda LA, Dion D, Dupuis G, Choinire M. A systematic literature review of 10 years of research on sex/gender and pain perception - part 2: do biopsychosocial factors alter pain sensitivity differently in women and men? Pain. (2012) 153:619-35. doi: 10.1016/j.pain.2011.11.026

2. Mogil JS. Sex differences in pain and pain inhibition: multiple explanations of a controversial phenomenon. Nat Rev Neurosci. (2012) 13:859-66. doi: $10.1038 / \mathrm{nrn} 3360$

3. Chapman CD, Benedict C, Schiöth HB. Experimenter gender and replicability in science. Sci Adv. (2018) 4:e1701427. doi: 10.1126/sciadv.1701427

4. Bartley EJ, Fillingim RB. Sex differences in pain: a brief review of clinical and experimental findings. Br J Anaesth. (2013) 111:52-8. doi: 10.1093/bja/aet127

5. Bernardes SF, Keogh E, Lima ML. Bridging the gap between pain and gender research: a selective literature review. Eur J Pain. (2008) 12:427-40. doi: 10.1016/j.ejpain.2007.08.007

6. Rolke R, Magerl W, Andrews Campbell K, Schalber C, Caspari S, Birklein F, et al. Quantitative sensory testing: a comprehensive protocol for clinical trials. Eur J Pain. (2006) 10:77-88. doi: 10.1016/j.ejpain.2005.02.003

7. Daniali H, Flaten MA. A qualitative systematic review of effects of provider characteristics and nonverbal behavior on pain, and placebo and nocebo effects. Front Psychiatry. (2019) 10:242. doi: 10.3389/fpsyt.2019.00242

8. Turk D, Monarch E. Biopsychosocial perspective on chronic pain. In: Turk DC, Gatchel RJ, editors. Psychological Approaches to Pain Management: A Practitioner's Handbook. 3rd ed. New York, NY: The Guildford Press (2018). p. 3-29.

9. Levine FM, Simone LL De. The effects of experimenter gender on pain report in male and female subjects. Pain. (1991) 44:69-72. doi: 10.1016/0304-3959(91)90149-R

10. Gijsbers K, Nicholson F. Experimental pain thresholds influenced by sex of experimenter. Percept Mot Skills. (2005) 101:803-7. doi: $10.2466 / \mathrm{pms}$.101.3.803-807

11. Keogh E. Gender differences in the nonverbal communication of pain: a new direction for sex, gender, and pain research? Pain. (2014) 155:1927-31. doi: $10.1016 /$ j.pain.2014.06.024

\section{DATA AVAILABILITY STATEMENT}

The raw data supporting the conclusions of this article will be made available by the authors, without undue reservation.

\section{ETHICS STATEMENT}

The studies involving human participants were reviewed and approved by Behavioral Research Ethics Board at the University of British Columbia. The patients/participants provided their written informed consent to participate in this study.

\section{AUTHOR CONTRIBUTIONS}

JM, LL, and JK designed and directed the project. NB and RB collected the data and ran the statistics, with assistance and supervision by JM and LL. Figures were created by JM and LL. JM wrote the manuscript, with all authors contributing to the final version.

\section{FUNDING}

JK was supported by a NSERC discovery grant.

12. Keogh E. The gender context of pain. Health Psychol Rev. (2020) 15:454-81. doi: 10.1080/17437199.2020.1813602

13. Quartana PJ, Campbell CM, Edwards RR. Pain catastrophizing: a critial review. Expert Rev Neurother. (2009) 9:745-58. doi: 10.1586/ern. 09.34

14. Arendt-Nielsen L, Yarnitsky D. Experimental and clinical applications of quantitative sensory testing applied to skin, muscles and viscera. J Pain. (2009) 10:556-72. doi: 10.1016/j.jpain.2009.02.002

15. Jutzeler CR, Sirucek L, Scheuren PS, Bobo T, Anenberg E, Ortiz O, et al. New life for an old idea: assessing tonic heat pain by means of participant controlled temperature. J Neurosci Methods. (2019) 321:20-7. doi: 10.1016/j.jneumeth.2019.04.003

16. Faul F, Erdfelder E, Lang AG, BuchnerA. A flexible statistical power analysis program for the social, behavioral, and biomedical sciences. Behav Res Methods. (2007) 39:175-91. doi: 10.3758/BF0319 3146

17. Aslaksen PM, Myrbakk IN, Høifødt RS, Flaten MA. The effect of experimenter gender on autonomic and subjective responses to pain stimuli. Pain. (2007) 129:260-8. doi: 10.1016/j.pain.2006.10.011

18. Kallai I, Barke A, Voss U. The effects of experimenter characteristics on pain reports in women and men. Pain. (2004) 112:142-7. doi: 10.1016/j.pain.2004.08.008

19. Weisse CS, Foster KK, Fisher EA. The influence of experimenter gender and race on pain reporting: does racial or gender concordance matter? Pain Med. (2005) 6:80-7. doi: 10.1111/j.1526-4637.2005.05004.x

20. World Medical Association. World Medical Association Declaration of Helsinki: ethical principles for medical research involving human subjects. J Am Med Assoc. (2013) 310:2191-4. doi: 10.1001/jama.2013.28 1053

21. Sullivan MJL, Bishop SR, Pivik J. The pain catastrophizing scale: development and validation. Psychol Assess. (1995) 7:524-32. doi: 10.1037/1040-3590.7.4.524

22. Vigil JM, Ba LNR, Alcock J, Maestes R. Laboratory personnel gender and cold pressor apparatus affect subject pain reports. Res Pain Manag. (2014) 19:e13-8. doi: 10.1155/2014/213950 
23. Greene LC, Hardy JD. Adaptation of thermal pain in the skin. J Appl Physiol. (1962) 17:693-6. doi: 10.1152/jappl.1962.17.4.693

24. Feine JS, Bushnell MC, Miron D, Duncan GH. Sex differences in the perception of noxious heat stimuli. Pain. (1991) 44:255-62. doi: 10.1016/0304-3959(91)90094-E

25. Bush FM, Harkins SW, Harrington WG, Price DD. Analysis of gender effects on pain perception and symptom presentation in temporomandibular pain. Pain. (1993) 53:73-80. doi: 10.1016/0304-3959(93) 90058-W

26. Otto MW, Dougher MJ. Sex differences and personality factors in responsivity to pain. Percept Mot Skills. (1985) 61:383-90. doi: 10.2466/pms.1985. 61.2 .383

27. Fillingim RB, Edwards RR, Powell T. The relationship of sex and clinical pain to experimental pain responses. Pain. (1999) 83:419-25. doi: 10.1016/S0304-3959(99)00128-1

28. Carter LE, Mcneil DW, Ma KEV, Ma JTS, Turk CL, Ries BJ, et al. Effects of emotion on pain reports, tolerance and physiology. Pain Res Manag. (2002) 7:21-30. doi: 10.1155/2002/426193

29. Thorn BE, Clements KL, Ward LC, Dixon KE, Kersh BC, Boothby JL, et al. Personality factors in the explanation of sex differences in pain catastrophizing and response to experimental pain. Pain. (2004) 20:275-82. doi: 10.1097/00002508-200409000-00001

30. Essick G, Guest S, Martinez E, Chen C, Mcglone F, Essick G, et al. Site-dependent and subject-related variations in perioral thermal sensitivity. Somatosens Mot Res. (2004) 21:159-75. doi: 10.1080/08990220400 012414

31. Jackson T, Iezzi T, Chen H, Ebnet S, Eglitis K. Gender, interpersonal transactions, and the perception of pain: an experimental analysis. J Pain. (2005) 6:228-36. doi: 10.1016/j.jpain.2004.12.004

Conflict of Interest: The authors declare that the research was conducted in the absence of any commercial or financial relationships that could be construed as a potential conflict of interest.

Publisher's Note: All claims expressed in this article are solely those of the authors and do not necessarily represent those of their affiliated organizations, or those of the publisher, the editors and the reviewers. Any product that may be evaluated in this article, or claim that may be made by its manufacturer, is not guaranteed or endorsed by the publisher.

Copyright (C) 2021 McDougall, Bailey, Banga, Linde and Kramer. This is an openaccess article distributed under the terms of the Creative Commons Attribution License (CC BY). The use, distribution or reproduction in other forums is permitted, provided the original author(s) and the copyright owner(s) are credited and that the original publication in this journal is cited, in accordance with accepted academic practice. No use, distribution or reproduction is permitted which does not comply with these terms. 